\title{
EL AGUJERO EN EL SABER
}

\section{ESTHELA SOLANO-SUÁREZ}

Psicoanalista en París. Licenciada en Psicología Universidad Nacional de Córdoba; DESS de psicología clínica y patológica Universidad Louis Pasteur Estrasburgo; DEA de Psicoanálisis Universidad de Paris VIII. Enseñante de la Sección Clínica, París-Saint-Denis. AME de la Escuela de la Causa Freudiana (ECF), de la New Lacanian School (NLS) y de la Asociación Mundial de Psicoanálisis (AMP). Autora de numerosos artículos en revistas especializadas.
Resumen: En su texto, "La enseñanza extracurricular o la fuga de Eros”, Germán García nos propone una lectura de la compleja relación del sujeto con el saber, para lo cual haciendo uso de su consabida erudición, avanza presentándonos diferentes estratos, diferentes niveles de lectura, diferentes aristas, con la finalidad de bordear el meollo de la cuestión.

Palabras Clave: Saber - Curiosidad sexual - Goce - Cuerpo - Síntoma

\section{EL SABER Y SUS ENREDOS}

En mi lectura de este texto, privilegiaré en primer lugar la referencia inicial de Germán García a propósito del aporte de Freud, relativo al niño y su relación con el saber.

Para Freud la aparición de la curiosidad sexual en la más temprana infancia no es otra cosa que un "empuje a querer saber" - Wissensdrang-1 empuje que proviene de un primer encuentro con lo sexual, es decir de aquello que se experimenta, que se siente en el cuerpo. Es en esa corriente erótica que el querer saber hunde sus raíces, precozmente e intensamente.
Abstract: : In La enseñanza extracurricular o la fuga de Eros, Germán García suggests a way of reading the complex subject-knowledge relation. His undoubted erudition allows him to show different layers, different reading levels and different perspectives to outline the core of the issue.

Key words: knowledge, sexual curiosity, enjoyment, body, symptom

Por ejemplo el pequeño Hans, que había comenzado a procurarse sensaciones agradables tocando su pequeño sexo, acordara a este órgano un vivo interés, a través de lo cual Hans, como lo señala Freud, se comporta como un verdadero "investigador."2 Ante esta solicitación erótica que se impone a partir del cuerpo se produce el despertar de una curiosidad, de un querer saber, de un cuestionamiento, de un interés, cuyo objetivo no es otro que el de dar un sentido a eso que se impone como experiencia de goce primitiva.

Podemos leer con Lacan cuál es la fuente traumática que despierta el interés y el querer saber del 
niño: para el pequeño Hans, "el goce que proviene de ese Wiwimacher -hace pipi- le es extranjero, al punto que esto está en el principio de su fobia”.3 También, en otro texto del mismo periodo Lacan enuncia: “¿En qué consiste la fobia del pequeño Hans? En el hecho de que él constata súbitamente que tiene un pequeño órgano que se mueve. Esto es perfectamente claro. Y él quiere darle un sentido. Pero, por más lejos que vaya ese sentido, ningún niño nunca siente que ese pene esté aferrado naturalmente. El considera siempre al pene como traumático. Quiero decir que él piensa que éste pertenece al exterior de su cuerpo. Por eso lo mira como algo separado, como un caballo que comienza a levantarse y a moverse." 4

Eso que se siente es inquietante, extranjero, y eso no es otra cosa que el goce. Si Lacan afirma que "la sexualidad es siempre traumática en tanto tal" 5 es porque el goce en tanto tal escapa al sentido, se impone en sí como héteros, es decir como exterior y extranjero a sí mismo, haciendo agujero en el saber.

\section{EL SABER ES LO QUE SE ARTICULA}

Antes de avanzar aclaremos el sentido del término "saber" en la conceptualización de Lacan.

El saber no es equivalente al conocimiento. Este último, según Lacan, participa de la confusión imaginaria suponiendo la dualidad de un sujeto y un objeto que se compenetran, se penetran, se adquieren, se poseen o se rechazan, se excluyen. Es una metáfora de la relación sexual, baste la expresión de la lengua "haber conocido, o no haber conocido mujer, y viceversa." El saber por el contrario es relativo a lo que se articula. ¿Qué es lo que se articula para los hablantes? Los significantes. Hablar, leer, escribir, disertar, discurrir, no es posible sino gracias al lenguaje. En el lenguaje los significantes, unidades sonoras propias de cada lengua, se articulan entre sí y abren a la dimensión de la significación, de aquello que se dice con la intención de querer significar algo. Lo que se quiere decir no es nunca unívoco, abre a todos los malentendidos e interpretaciones. Retengamos solamente que la articulación mínima para que el lenguaje produzca un efecto de sentido es la de un significante S1 con otro significante S2. Por eso Lacan se sirve de la escritura S2 como siendo la escritura del saber.

Si la sexualidad es traumática lo es para los seres hablantes, ya que el lenguaje los ha separado de un saber hacer instintual con respecto a lo sexual. En el cuerpo, o mejor dicho en el nudo entre el inconsciente y el cuerpo, la sexualidad se inscribe en ruptura con una ley "natural". En el reino animal por el contrario, el saber hacer impone una rutina regulada de los encuentros sexuados para la reproducción de la especie.

Ninguna educación sexual puede suplir a ese saber que no hay, que no se inscribe, que hace agujero en el saber inconsciente.

En un análisis se puede descifrar un saber, el que la lengua inscribe en términos de inconsciente, pero el límite de ese saber es lo que jamás se podrá incluir en él para los hablantes sexuados: saber cómo dos cuerpos hagan Uno en el goce.

\section{LOS DESTINOS DEL "EMPUJE DE SABER"}

Retomemos el punto del cual partimos: la experiencia sexual infantil traumática y el despertar del empuje de saber. En su investigación solitaria el niño avanza solo hacia una elucubración de saber y construirá ficciones fantasmáticas a las cuales Freud da el nombre de teorías sexuales infantiles.6 Estas "teorías" pueden elucidarse en un análisis como siendo constitutivas de los fantasmas inconscientes los cuales rigen los modos de orientación sexual de cada ser hablante y hacen consistir una versión del imposible "rapport" entre dos seres sexuados.

Un aporte importante que podemos retener de Freud a este respecto es el de indicarnos que la investigación sexual infantil como primera tentativa de autonomía intelectual, fracasará ante lo real, es decir el sin sentido proveniente de lo sexual. Así Freud distingue tres destinos posibles del empuje al saber, provenientes de la conexión precoz con el interés por lo sexual.7

En el primer caso de esta configuración, la avidez de saber sucumbe a la fuerza de la represión, produciendo una inhibición neurótica con respecto al saber, limitando así toda actividad intelectual.

En el segundo caso descripto por Freud, se trata de la imposición compulsiva de la rumiación intelectual la cual proviene del retorno de un querer saber infantil reprimido. En este caso el pensamiento se sexualiza y se convierte en fuente de goce como también de angustia, naufragando, como la investigación sexual infantil, en la imposibilidad de llegar a una conclusión. Es el caso típico del síntoma que caracteriza la neurosis obsesiva.

El tercer destino del empuje de saber, el más raro 
como también el más perfecto -en el cual Freud identifica la posición de Leonardo Da Vinci - el saber escapa a la inhibición del pensamiento gracias a la sublimación de la pulsión, lo cual permite que el destino de la pulsión se asocie a la avidez de saber, pudiendo entonces el sujeto servirse del empuje pulsional, poniéndolo al servicio del interés intelectual.

De lo que precede podemos atisbar que la relación con el saber para cada uno es singular y esta entretejida a las peripecias de la relación del sujeto a la pulsión, es decir a las resonancias dejadas en el cuerpo por un decir.

\section{ELOGIO DEL ENCUENTRO}

Como Germán García lo señala en su texto, para Freud había tres dimensiones sociales imposibles: gobernar, educar y psicoanalizar. Imposibles no quiere decir que debemos sucumbir a la impotencia, sino por el contrario, saber cuál es la imposibilidad en juego para, con el recurso del discurso, encontrar una orientación.

En este sentido Lacan formalizó los matemas de los discursos, 8 distinguiendo así el discurso del amo, el discurso universitario, el discurso histérico, el discurso del analista, a los cuales agregara luego el discurso de la ciencia y el discurso capitalista. Esta fue la respuesta de Lacan al mayo del 68 francés. No entraré aquí en el desarrollo de cada uno de los discursos. Solo indicaré que en cada uno de ellos el saber, en tanto S2, se encuentra en lugares diferentes. En el discurso del analista, es decir en el lazo social que fundamenta la experiencia de un análisis entre el analista y el analizante, el saber ocupa el lugar de la verdad. Es el único discurso en el cual la articulación de saber puede venir en el lugar de la verdad variable, tal como se produce en un análisis.

En el discurso universitario por el contrario, el saber ocupa el lugar del agente, el lugar que comanda. Es necesario en un dispositivo curricular acceder a ciertos saberes, cuya adquisición calificara profesionalmente a quien, al final del recorrido, adquirirá un diploma validado por el Estado.

La adquisición de una competencia profesional reconocida socialmente requiere un recorrido curricular. En la época en que cursé estudios de psicología, en la Universidad Nacional de Córdoba, en los años 60 , hacía poco tiempo que esta carrera existía y tuve la gran suerte de iniciarme en ella a la lectura de Freud y autores post freudianos, como también al estudio de las principales corrientes fi- losóficas. Si bien en el programa contaban las estadísticas como la neurofisiología, lo esencial de aquella orientación eran las Humanidades. Hoy el panorama de la psicología en los principales países europeos se orienta cada vez más hacia un abordaje comportamentalista y experimental. Las neurociencias reinan. El inconsciente y la psicopatología de inspiración psicoanalítica van siendo expulsados de los programas. Como es el caso hoy en día en Francia en las nuevas proposiciones para el programa de Filosofía de los bachilleres: los conceptos de "inconsciente" y de "trabajo" no estarán ya incluidos, con lo cual Freud y Marx son deportados fuera del recorrido curricular.

Lo cual indica que "los saberes" se distribuyen según la orientación ideológica dominante.

Esto no impide el "encuentro", feliz encuentro que podemos experimentar en nuestras vidas con un autor, con un libro, con un texto, con un docente, quien nos abrirá las puertas de un saber inédito. Ese fue mi caso y no creo que sea una excepción. El encuentro que me abrió las vías de la experiencia analítica y del encuentro con Lacan específicamente, fue un encuentro extra curricular.

Y la experiencia analítica me sirvió para saber que devenir analista no es posible fuera de la experiencia de un análisis. No hay formación curricular del analista, porque no releva de una competencia universitaria, ni de una calificación relativa a un diploma.

Analista es aquel que resulta de su experiencia de analizante. Es una posición subjetiva que deriva de la reducción del goce del síntoma gracias a una elaboración de saber sostenida hasta el punto en que se demuestra su imposibilidad. De lo cual resulta lo irreductible del goce fuera de sentido del sinthome, que no se atraviesa, que es del orden del Uno que marca lo más singular de la relación del cuerpo con lalengua.

\section{LA CREENCIA Y EL SABER}

La conquista de un saber que no se sabe, en el psicoanálisis tiene como eje el dispositivo de la transferencia. Este comporta que quien demanda un análisis supone que aquello de lo cual sufre, su síntoma "quiere decir algo", algo que él no sabe. Lacan estudiando la transferencia recurre al ejemplo de El Banquete de Platón. Alcibíades, supone que Sócrates contiene en sí un objeto maravilloso, el agalma del saber, y que éste no acepta sucumbir a la solicitación amorosa de Alcibíades 
que desea que Sócrates le ceda este objeto. La ilusión de la suposición de saber es atribuida al otro, cuando en realidad el saber del que se trata no es otra cosa que el saber que debemos aprender a leer y que es relativo al inconsciente. Pero aquel a quien le suponemos un saber, lo amamos. La conquista del saber se saldará en un análisis, no por la increencia sino por la caída del analista del lugar de supuesto saber. Lo cual no quiere decir que se descrea en el psicoanálisis, sino que la transferencia se desplaza hacia la comunidad analítica, como transferencia de trabajo en la que cada uno se hace responsable del destino y del progreso del psicoanálisis.

Amor, creencia y suposición de saber se conjugan. La increencia que Freud calificó como propia de la posición del paranoico, la Unglauben, es signo de la forclusión del saber inconsciente.

\section{EL DERRUMBE DE LA AUTORIDAD}

El discurso de la ciencia en su conjugación con el discurso capitalista han producido como consecuencia el declive, y quizá el derrumbe del Padre y del orden social que reinaba acorde a su función. Uno de los efectos de esta disolución es la crisis de la autoridad, la progresiva evaporación de las estructuras jerárquicas y de la predominancia de modelos verticales, como lo señala Germán García en su texto.

En consecuencia Germán indicaba los nuevos modos de relación con el saber que derivan del uso de las redes sociales y de Internet. Un saber al alcance de la mano, inagotable, disponible y sin relación con una "autoridad" que lo dispense en el marco de un recorrido curricular. Como Germán lo indica con gran pertinencia, en este nuevo modo de consumo domina un cierto autismo del goce, con la paradoja que implica lo virtual de excluir el "cuerpo a cuerpo" tanto a nivel sexual como a nivel del consumo de saberes.

Es nuestro siglo XXI, no podemos lamentar lo que fue, hay que hacer con lo que hay. Las nuevas tecnologías, todos los aparatos, los gadgets, que constituyen ahora nuestra ortopedia cotidiana, solo ponen de manifiesto las nuevas formas del malestar de la civilización, como Freud ya lo indicó en su momento. Son instrumentos derivados de la ciencia y desarrollados por la tecnología, que están a nuestra disposición para hacer uso de ellos. Introducen nuevos modos de lazo social, nuevos modos de relación con el saber, sin que por lo tanto impliquen la desaparición del imposible relativo al saber.

\section{LA POLÍTICA DEL SINTHOME}

Los síntomas siguen, para cada uno, marcando la emergencia de lo real como imposible. El síntoma es lo que se pone al cruce, lo que obstaculiza la marcha imperiosa de la exigencia de rentabilidad y de éxito del amo contemporáneo. Las nuevas vías de difusión que nos procuran los gadgets, pueden ser propicias para producir un encuentro inusitado con otro discurso, el psicoanalítico, el único que se ocupa debidamente de lo que hace síntoma para cada hablante y del cual se puede extraer un saber en posición de verdad.

El psicoanálisis enuncia en cierto modo: Tu puedes saber por qué no eres como todos, puedes saber cuál es tu singularidad con respecto al goce y al saber y desde ahí, saber hacer con tu existencia, que no es otra que la de tu "sinthome".

\section{Notas}

1. Freud S. : "Las teorías sexuales de los niños", (1908), Biblioteca Nueva, Vol. I, Madrid 1967. p. 1172.

2. Freud S. : "Historias clínicas, Análisis de la fobia de un niño de cinco años”, Biblioteca Nueva, Vol. II, p. 698.

3. Lacan J., "Conférence à Genève sur le symptôme" ", "La Cause du désir n 95, Navarin éditeur", 017, p. 14.

4. Lacan J.: "Conferencias y entrevistas en las universidades norte -americanas”, noviembre-diciembre 1975, Scilicet 6/7, Seuil, 1976, p. 22.

5 Ibidem.

6 Freud S.: "La sexualidad infantil. La investigación sexual infantil", op. citado, Vol I, p.799.

7 Freud S.: "Un recuerdo infantil de Leonardo de Vinci", op. citado, Vol II, p. 403 y siguientes.

8 Lacan J.: "El Seminario Libro XVII, El reverso del psicoanálisis", 19691970, Paidós, Buenos Aires, 1992. 